Orozco Cazco, G.H., Tejedor Tejedor, F. y Calvo Álvarez, M.I. (2017). Meta-análisis sobre el efecto del software educativo en alumnos con necesidades educativas especiales. Revista de Investigación Educativa, 35(1), 35-52 DOI: http://dx.doi.org/10.6018/rie.35.1.240351

\title{
Meta-Análisis sobre el efecto del Software Educativo en alumnos con Necesidades Educativas Especiales
}

\section{Meta-Analysis in the efect of Educative Software on students with Special Educational Needs}

\author{
Gustavo Homero Orozco Cazco, Francisco Javier Tejedor Tejedor y María Isabel Calvo Álvarez \\ *Universidad Nacional de Chimborazo \\ **Universidad de Salamanca \\ *** Universidad de Salamanca
}

\begin{abstract}
Resumen
El objetivo de este trabajo fue determinar el efecto que produce el uso del Software Educativo en alumnos de 6 a 12 años con Necesidades Educativas Especiales (NEE). Como metodología se utilizó el Meta-análisis, que permitió integrar cuantitativamente los resultados de las investigaciones primarias del problema planteado de forma objetiva y sistemática, para lo cual se seleccionaron 10 estudios mediante criterios de inclusión y exclusión, los mismos que produjeron un total de 14 tamaños de efecto independientes. Se obtuvo como resultados una media del tamaño de efecto $d+=1.22$, y una estimación puntual del tamaño de efecto $d .=.21$, siendo un valor significativo bajo de la incidencia del Software Educativo en apoyo a las NEE. En conclusión, es importante resaltar la necesidad de continuar investigando sobre Tecnologías de la Información y Comunicación enfocadas a solventar las dificultades del aprendizaje que se producen en las aulas para garantizar una verdadera escuela inclusiva.

Palabras clave: software educativo; tecnologías de la información y comunicación (TIC); necesidades educativas especiales; educación especial.
\end{abstract}

Correspondencia: Gustavo Homero Orozco Cazco, gorozco@unach.edu.ec, Av. Eloy Alfaro s/n y 10 de Agosto, 060104 Riobamba, Ecuador. 


\begin{abstract}
The objective of this work was to determine the effect that the uses of the Educative software produce in the students of 6 to 12 years old with Special Educational Needs (NEE). The methodology applied was the Meta-analysis; it allows to integrate in a qualitative way the results of the primary investigarions about the problem in an objective and systemic. For this purpose, 10 study cases were selected trough inclusion and exclusion process, with a total of 14 independents effects. It got a result of a media of effect $d+=1.22$, a punctual estimation of effect of $d .=21$, being a significantly low value of the incidence of the educative Software in support to the NEE. In conclusion, it is important to highlight the need of more investigation about the Communicative and Informative Technologies focused on solving the difficulties of the learning in the classroom to guarantee a truly inclusive school.

Keywords: educative software; informative and communicative technologies (ICT); special educative needs; special education.
\end{abstract}

\title{
Introducción
}

La irrupción de las Tecnologías de la Información y Comunicación (TIC) ${ }^{1}$ en la sociedad actual, sin duda está modificando los procesos educativos en todos los niveles, provocando una nueva revolución, la del conocimiento (Cebrián, 2011), que obliga de algún modo a configurar de distinta manera los aspectos sociales, culturales y económicos de las instituciones, con el objetivo de mejorar la interacción y comunicación de los diferentes estamentos para crear un pensamiento colectivo (Cabero, 2008), siendo necesario para ello contar con materiales y recursos innovadores (en lo posible diseñados y producidos por la persona que va a aplicar) que coadyuven a un aprendizaje activo (Amar, 2013).

Dentro de las TIC, se encuentra un medio didáctico que se ha ido incorporando paulatinamente en los centros educativos como es el Software Educativo ${ }^{2}$, que según García-Valcárcel y Hernández (2013) crean entornos de aprendizaje flexibles e interactivos, contribuyendo con el desarrollo cognitivo y motivacional de los estudiantes. Sin embargo, este recurso (Software Educativo) todavía es muy limitado en cuanto al apoyo que requieren los estudiantes, sobre todo los alumnos con Necesidades Educativas Especiales (NEE), por cuanto la masificación es más lucrativa que realizar un producto para un caso en concreto, siendo necesario recurrir a lo gratuito o libre para realizar adaptaciones que cubran las dificultades del aprendizaje que se producen en el aula (Hervás \& Toledo, 2007).

Cabe mencionar que durante varias décadas se ha ido modificando y evolucionado la terminología, clasificaciones y taxonomías sobre las personas con dificultades de

1 TIC es el conjunto de tecnologías (ordenadores, consolas de videojuegos, robótica, tabletas digitales, smartphone, software educativo y demás), que permiten la adquisición, producción, almacenamiento, tratamiento, comunicación y presentación de información en forma de voz, imágenes, y otros (García-Valcárcel, 2003, p. 42).

2 Software Educativo son programas para ordenador creados con la finalidad específica de ser utilizados como medio didáctico, es decir para facilitar los procesos de enseñanza y de aprendizaje (Marqués, 1995, p. 20). 
aprendizaje, con el propósito de comprender la problemática y así poder erradicar la discriminación que se hace presente en las aulas, evitando etiquetaciones hacia los alumnos de acuerdo a deficiencias o discapacidades (Marín, 2004).

La atención a las personas que presentaban limitaciones o retraso en el contexto escolar derivadas de deficiencia o discapacidad ha pasado por distintas etapas y denominaciones.

Por tanto, el concepto “Necesidades Educativas Especiales", aparece en los años 60, recogido por primera vez en el Informe Warnok de 1978, con la finalidad de transformar el enfoque de la escuela tradicional, centrada en el déficit, a una escuela integradora, centrada en las necesidades del alumno. El Informe recoge como las necesidades educativas pueden ser temporales o permanentes durante su etapa escolar, dando lugar a una diversidad de alumnado que requiere de una atención individualiza para responder a sus necesidades, pero en un mismo ambiente social, el fin es la inclusión del alumno en el contexto escolar y social (ARARTEKO, 2001; Calvo \& Verdugo, 2012; Marchesi, 2014; Parra, 2010; Sanz del Río, 1996).

Desde entonces, se han producido una infinidad de definiciones sobre el concepto "Necesidades Educativas Especiales" por su complejidad y variedad de perspectivas (médicas, psicológicas, pedagógicas, sociológicas y demás), como son las de Bautista (2002), González (1996), Ricoy y Feliz (2002), citándose para este trabajo la que señala el Boletín Oficial del Estado-España (2006, p. 52), "se entiende por alumnado con Necesidades Educativas Especiales, aquel que requiera, por un período de su escolarización o a lo largo de toda ella, determinados apoyos y atenciones educativas específicas derivadas de discapacidad o trastornos graves de conducta".

Como en el caso de las definiciones, también han surgido varias clasificaciones sobre el alumnado con NEE, cada autor se ha centrado en un aspecto, como es el caso de Cerdá (1990), Cortés (1999), entre otros, tomándose como referencia la clasificación que propone López y Valenzuela (2015, p. 43): NEE Permanentes (discapacidad intelectual, discapacidad sensorial, discapacidad motora,...) y NEE Transitorias (trastornos emocionales, trastornos conductuales, trastornos específicos del lenguaje, trastorno de déficit de atención e hiperactividad,...).

Entonces, hablar de alumno con NEE y de alumno con discapacidad conlleva entender la educación como un derecho y pasar de una educación especial y segregada a una educación inclusiva, lo que obliga una transformación de las escuelas del currículum, de la formación del profesorado, de las metodologías y de las actitudes de todos los implicados y por ello también de las políticas educativas; es dar respuesta a todos independiente de sus necesidades y características individuales y/o sociales. Hablar en el siglo XXI de alumnos con discapacidad y TIC, es hablar de inclusión educativa es ofrecer al alumnado una educación de calidad, en la que todos se beneficien de una educación que parte de sus necesidades, valora la diversidad y respeta las diferencias individuales. "La Educación Inclusiva no es una cuestión marginal, sino que va a ser crucial para lograr una educación de calidad de todo el alumnado y para el desarrollo de sociedades más inclusivas" (Organización de las Naciones Unidas para la Educación, la Ciencia y la Cultura [UNESCO], 2008, p. 5).

Doval (2011), manifiesta que si no hay una adecuada implementación de las TIC seguirá produciéndose la exclusión social y aumentando la brecha digital, por lo que 
sugiere la realización de una adecuada evaluación de la accesibilidad y la competencia digital del alumnado, un proceso de reflexión y toma de decisiones sobre la idoneidad de las TIC, y su incorporación con las debidas adaptaciones del alumno, ya que las ventajas de las TIC, según Cabero, Fernández y Córdoba (2007) son diversas dependiendo del tipo de discapacidad (Ayudan a superar sus limitaciones, favorecen la autonomía y comunicación, facilitan la construcción del aprendizaje, adaptación a su ritmo de aprendizaje, evitan la marginación, entre otras).

Ante lo mencionado, es imperioso indicar, el rol que debe asumir el profesorado en la incorporación de las TIC para facilitar la inclusión de alumnos con NEE, ya que se requieren procesos de enseñanza-aprendizaje innovadores para alcanzar mejoras sustanciales en la educación, con lo cual se pueda atender a la diversidad del alumnado (Marín \& Latorre, 2007, p. 270). Sin embargo, las mismas autoras manifiestan la necesidad de "reestructurar la cultura, las políticas y las prácticas de las escuelas" para cumplir con una escuela para todos, coincidiendo en parte con lo que dicen González y De Pablos (2015) sobre la importancia que tienen las instituciones para implantar cambios pedagógicos con la combinación de las TIC.

Por esta razón, es pertinente citar las consideraciones que hacen Luque y Rodríguez (2009, p. 6) para lograr la igualdad de oportunidades en alumnos con NEE y poder tomarlas en cuenta para acciones futuras:

1) Integración de la tecnología en el aula para alumnos con discapacidad; 2) Fomento de ayudas para la adquisición de equipamiento específico y ayudas técnicas de acceso al ordenador por el Centro; 3) Formación del profesorado en Software Educativo para alumnos con NEE; 4) Formación en criterios de diseño universal, pautas de accesibilidad en el diseño de páginas web; 5) Investigación sobre la metodología y didáctica de la aplicación de las TIC en el alumnado con discapacidad; 6) Colaboración entre profesionales (profesorado, productores de software, directores de centros, etc.) a través de redes regionales y nacionales, así como entre profesionales y padres de estos alumnos; y 7) Sensibilización social sobre la importancia de la accesibilidad para la educación de este grupo de personas (alumnos con NEE); y 8) Fomento de la cultura del Diseño para Todos, así como de la investigación de equipos adaptados y ayudas técnicas.

En la misma línea, Cabero y Córdoba (2009, p. 74) habla de tres aspectos para favorecer la inclusión digital y atender la diversidad del alumnado en la escuela, los cuales coinciden en parte con algunos puntos del apartado anterior (1. Facilitar la presencia y acceso a las TIC; 2 . Revisar sus características de adaptabilidad y accesibilidad; y 3. Alfabetización digital).

\section{Objetivos}

En el presente trabajo se utilizó la metodología del Meta-análisis con el fin de determinar el tamaño del efecto que se ha producido con la aplicación del Software 
Educativo en alumnos con Necesidades Educativas Especiales, siendo relevante por cuanto nos permitió realizar un análisis estadístico de los resultados de investigaciones primarias relativamente homogéneas de forma rigurosa (Glass, 1976) para futuros estudios en la misma línea de investigación.

Para ello, se plantearon algunas interrogantes que permitieron dilucidar la temática planteada, las mismas que citan a continuación: ¿Cuáles son las NEE más apoyadas por los investigadores mediante el Software Educativo?, ¿Cuál es el área o asignatura que fue beneficiada con el uso del Software Educativo para superar las dificultades del aprendizaje de los alumnos con NEE?, ¿Cuáles son las funciones del Software Educativo aplicadas por los investigadores en el diseño del medio didáctico para mejorar el proceso de enseñanza de los alumnos con NEE?, y por último ¿Cuál es el tamaño del efecto estimado en el conjunto de resultados de los estudios primarios encontrados?

De los cuestionamientos descritos anteriormente se formularon cuatro objetivos: 1) Identificar las investigaciones de Software Educativo aplicado a niños de entre 6 y 12 años de edad con NEE; 2) Integrar cuantitativamente los resultados obtenidos en la investigaciones seleccionadas en cuanto al efecto del Software Educativo aplicado a niños con NEE; 3) Establecer el nivel de heterogeneidad de los estudios localizados; y 4) Determinar la incidencia del Software Educativo en niños de entre 6 y 12 años con NEE en diferentes áreas del conocimiento.

\section{Metodología}

Como se indicó anteriormente, en este trabajo se aplicó una Revisión Meta-analítica, en la cual se deben cumplir con una serie de tareas para encontrar el tamaño del efecto de un conjunto de estudios, las mismas que se describen a continuación:

\section{Búsqueda de la literatura}

La búsqueda de información se desarrolló con un alto grado de rigurosidad y delicadeza como indica Botella y Gambara (2002), para evitar en gran medida el sesgo que puede provocar el procedimiento elegido. En tal sentido, se plantearon ciertos criterios de inclusión y exclusión que sugieren Durkay y Lipsey (1991), acorde a las preguntas de investigación y de esta manera también delimitar la población de estudios para su posterior evaluación.

Los criterios de inclusión que debieron cumplir los estudios fueron: 1) Que hayan sido desarrollados en el período 2004 - 2014; 2) Haber utilizado como metodologías de investigación la Cuasi-experimental, Pre-experimental y Estudio de Casos; 3) Los estudios muestren evidencias de resultados que permitan determinar el tamaño del efecto; 4) Los estudios deben tener una población o muestra con niños de entre 6 y 12 años de edad; y 5) El idioma a tomar en cuenta para seleccionar el estudio fueron el español y el inglés. Y los de exclusión: 1) Los estudios que no incluyan la edad cronológica y cognitiva de la población o muestra de los niños que se encuentren en el rango de 6 a 12 años; y 2) Los estudios que no indiquen el tipo de aplicación 
del Software Educativo en el apoyo de las NEE. Estos criterios al final permitieron seleccionar 10 trabajos de investigación que dieron lugar a 14 estudios independientes, además de un protocolo de evaluación.

En cuanto al procedimiento de búsqueda, se desarrolló en varios canales o fuentes de información como menciona Cooper (1994) citado por Botella y Gambara (2002): Canales Informales (Vía, en la cual se puede localizar una gran cantidad de información): Google Académico; Canales Formales Primarios (Vía, en la cual se cual se pueden localizar estudios que han sido revisados por comités científicos): Journal of Computer Assisted Learning; y Canales Formales Secundarios (Vía, en la cual se pueden localizar estudios primarios de forma indirecta): WOS, Scopus y Eric. Además, se utilizó varias combinaciones de palabras claves para alcanzar un nivel de éxito aceptable en la exploración de resultados sobre investigaciones con referencia al objeto de estudio como sugiere Hernández (2009): Software Educativo, Programas informáticos Educativos, Programas de Enseñanza asistido por ordenador, Educación Especial, Necesidades Educativas Especiales, Niñ ${ }^{*}, C^{*}$ Child ${ }^{*}$, Educative Software, Computer Assisted Instruction, Computer Assisted Learning, Special Education y Need Education.

Además, es importante señalar que el proceso de búsqueda se realizó en el mes de febrero de 2015, obteniéndose una cantidad considerable de resultados por la variedad de combinaciones de palabras claves empleadas en cada una de las fuentes de información (ver tabla 1). Para el primer filtrado, nos limitamos a la revisión del título y en algunos casos en el resumen del trabajo de investigación, lo cual permitió seleccionar 147 estudios.

Luego de haber reducido la cantidad de resultados, los estudios seleccionados fueron sometidos a una valoración de acuerdo a los criterios de inclusión y exclusión propuestos en este trabajo, provocando una disminución significativa a 38 estudios.

Por último, los 38 estudios seleccionados fueron repartidos entre los responsables del presente trabajo para su respectiva evaluación, porque la calidad de un meta-análisis depende de los estudios elegidos. Para ello, se utilizó un protocolo de evaluación que contenía 12 elementos (claridad y estructura del título, revisión de la literatura, rigor metodológico, citas actualizadas,...), con una escala de valoración de 0 a 5 . Sin duda esta fue la etapa más laboriosa por su importancia, obteniéndose de este proceso la selección de nueve artículos (Alcaraz, Ballesta \& Lozano, 2011; Atkins, Chakpitak, Sharp, Sureephong, \& Wicha, 2012; Contreras \& Contreras, 2011; Cullen, Richards, \& Lawless, 2008; Gómez \& Ortega, 2006; Hetzroni \& Tannous, 2004; Tan \& Mei Lin, 2012; Sánchez \& Elías, 2007; Simion \& Folostina, 2013) y una tesis doctoral (Ventura, 2012) (un artículo de los seleccionados constaba tanto en WOS como en SCOPUS), estudios que finalmente son parte de este meta-análisis.

En este punto cabe indicar, que los trabajos eliminados durante el proceso descrito, correspondían a estudios enfocados al diseño, desarrollo, implementación, uso, ejecución y aplicación de Software Educativo para el apoyo a niños, pero sin la información necesaria que se requiere para codificar de acuerdo a las características definidas en este tipo de trabajos. 
Tabla 1

Resultados de la búsqueda en los canales de información

\begin{tabular}{|c|c|c|c|c|c|}
\hline \multirow{3}{*}{ Canal de Información } & \multirow{2}{*}{\multicolumn{2}{|c|}{$\begin{array}{l}\text { Resultados de la } \\
\text { Búsqueda } \\
\text { Idioma }\end{array}$}} & \multirow{2}{*}{\multicolumn{2}{|c|}{$\begin{array}{c}\text { Estudios Selecciona- } \\
\text { dos } \\
\text { Idioma }\end{array}$}} & \multirow{3}{*}{$\begin{array}{l}\text { Fechas de } \\
\text { Búsqueda }\end{array}$} \\
\hline & & & & & \\
\hline & Español & Inglés & Español & Inglés & \\
\hline Google Académico & 644 & 1950 & 3 & 4 & $03 / 02 / 2015$ \\
\hline $\begin{array}{l}\text { Journal of Computer } \\
\text { Assited Learning }\end{array}$ & 0 & 61 & 0 & 1 & $16 / 02 / 2015$ \\
\hline WOS & 0 & 67 & 0 & 1 & $18 / 02 / 2015$ \\
\hline ERIC & 0 & 66 & 0 & 1 & $23 / 02 / 2015$ \\
\hline SCOPUS & 1 & 127 & 0 & 1 & $26 / 02 / 2015$ \\
\hline
\end{tabular}

\section{Codificación de los estudios}

Las características seleccionadas para un meta-análisis no deben ser elegidas al azar, por tal razón se siguió la estructura que propone Lipsey (1994) para conservar el rigor que exige esta metodología, la misma que se divide en tres aspectos: 1) Sustantivos: Lugar del estudio, Necesidades Educativas Especiales, Edad, Grado o Nivel Educativo, Asignatura, Duración de aplicación, Software utilizado y Función del Software; 2) Metodológicos: Tipo de Diseño, Muestra, Instrumento y Técnica de Análisis; y 3) Factores Extrínsecos: Autor (s), Tipo de Documento, Año de Publicación, Fuente de Publicación e Idioma.

Para garantizar la fiabilidad de la base de datos codificada de las características detalladas en el párrafo anterior, se utilizó la Kappa de Cohen entre dos expertos, para lo cual se plantearon dos hipótesis $\left(\mathrm{H}_{0}=0\right.$ No existe acuerdo entre los expertos; $\mathrm{y} \mathrm{H}_{1}=1$ Existe acuerdo entre los expertos) que validamos mediante el SPSS V.20.

En tal sentido, se evaluaron 34 categorías, de las cuales se registró un 100\% de casos válidos, es decir que todos los ítems puestos a consideración fueron observados por los expertos. De ahí, nos permitió establecer el nivel de acuerdo entre los expertos que evaluaron la codificación de los estudios, alcanzando un $76.5 \%$ de acuerdo y un $23.5 \%$ en desacuerdo, que según Landis y Koch (1977) citado por Botella y Gambara (2002), es un Acuerdo Sustancial (Relativamente Satisfactorio) por estar dentro del rango 0,61-0,80, provocando una mínima modificación en la Base de Datos definitiva con la supresión de tres categorías: Horas de Aplicación del Software Educativo, Disciplina del Autor y Soporte o Medio Tecnológico.

\section{Cálculo y análisis del tamaño del efecto}

Para determinar el nivel de significancia o el tamaño del efecto de acuerdo a las preguntas de investigación, se procedió en primer lugar a transformar todos los resultados de los estudios primarios seleccionados a una medida común (métrica común), 
por la variedad de información que presentan los investigadores en los informes finales, tal como indica Botella y Gambara (2002). En segunda instancia, se calculó el tamaño del efecto utilizando el índice "diferencia de medias estandarizada" ( $\boldsymbol{d}$ de Cohen), que representa el grado de eficacia que ha tenido el Software Educativo en las distintas Necesidades Educativas Especiales cubiertas en los niños de 6 a 12 años.

En las tablas 2 y 3 se describen los resultados obtenidos, debiéndose indicar que los unos corresponden a los diseños intra-grupo (pre-test y post-test), definiéndose $d$ como la diferencia entre la media del pre-test y la media del post-test dividido por la desviación típica intra-grupo conjunta; y los otros, a los diseños inter-grupos, donde $\mathrm{d}$ se definió con $\boldsymbol{d}=\boldsymbol{d}^{E}-\boldsymbol{d}^{C}$, siendo $\boldsymbol{d}^{E}$ y $\boldsymbol{d}^{C}$, la diferencia de medias tipificadas entre el pre-test y el pos-test de los grupos experimental y de control, respectivamente como indican Sánchez, Olivares y Rosa (1998).

Tabla 2

Resultados obtenidos con los estudios intra-grupo

\begin{tabular}{cccccc}
\hline Diseño & Artículo & $\begin{array}{c}\text { Varianza } \\
\mathbf{S}^{2}\end{array}$ & $\begin{array}{c}\text { Desviación } \\
\text { Típica } \\
\text { S }\end{array}$ & $\begin{array}{c}\text { Diferencia } \\
\text { de medias } \\
\mathbf{d}\end{array}$ & $\begin{array}{c}\text { Correlación } \\
\text { de Pearson } \\
\mathbf{r}\end{array}$ \\
\hline & 1 & 153.46 & 12.39 & 4.33 & .91 \\
& 2.1 & 4.80 & 2.19 & 2.69 & .80 \\
Intra-grupo & 2.2 & 10.91 & 3.30 & 1.92 & .69 \\
& 3 & .9 & .95 & 2.03 & .71 \\
& 4 & 18.85 & 4.34 & 1.45 & .59 \\
& 7.1 & 9.34 & 3.06 & -.41 & .20 \\
& 7.2 & 2.94 & 1.71 & 0.58 & .28 \\
& 9.1 & 3.45 & 1.86 & .28 & .14 \\
& 9.2 & 2.83 & 1.68 & 1.11 & .49 \\
& 10 & 1.44 & 1.20 & -.20 & .10 \\
\hline
\end{tabular}

Tabla 3

Resultados obtenidos con los estudios inter-grupo

\begin{tabular}{lcccccccccccc}
\hline & \multicolumn{1}{c}{ Grupo Experimental } & \multicolumn{7}{c}{ Grupo Control } \\
\hline Diseño & Artículo & $\mathbf{n}_{\mathrm{E}}$ & $\mathbf{N}_{\mathrm{C}}$ & $\mathbf{t}$ & $\mathbf{d}_{\mathrm{E}}$ & $\mathbf{n}_{\mathrm{E}}$ & $\mathbf{N}_{\mathrm{C}}$ & $\mathbf{t}$ & $\mathbf{d}_{\mathrm{C}}$ & $\mathbf{d}$ & $\mathbf{r}$ \\
& 5.1 & 90 & 90 & 7.88 & .16 & 90 & 90 & .67 & .01 & .15 & .22 \\
& 5.2 & 90 & 90 & 3.25 & .07 & 90 & 90 & .98 & .02 & .05 & .03 \\
$\begin{array}{l}\text { Inter- } \\
\text { grupo }\end{array}$ & 6 & 9 & 9 & 2.95 & 1.38 & 9 & 9 & 1.46 & .68 & .70 & .33 \\
& 8 & 9 & 8 & 5.81 & 2.85 & 9 & 8 & .79 & .39 & 2.46 & .77 \\
\hline
\end{tabular}


En los resultados obtenidos anteriormente se observa dos tamaños del efecto que registran un dato negativo, y los doce restantes datos positivos, prediciendo de esta manera que el cálculo de la estimación combinada será un valor distinto de cero.

Además es necesario indicar que la $d$ de Cohen y la Correlación de Pearson ( $r$ ), nos permitió calcular mediante la técnica estadística Estimación Combinada del Efecto para contestar a las preguntas que dirigen el análisis de datos de un meta-análisis ¿Cuál es el tamaño del efecto que podemos estimar a partir de los resultados encontrados? y ¿Los resultados encontrados en los estudios primarios son lo suficientemente heterogéneos como para que esa dispersión necesite variabilidad? como indica Botella y Gambara (2002).

\section{Resultados}

En este apartado se presenta un resumen del análisis descriptivo desarrollado con los datos obtenidos de los estudios seleccionados con las variables o características elegidas previamente.

\section{Estudio descriptivo de las investigaciones}

Los documentos seleccionados para el meta-análisis en un 90\% se encontró en forma de artículo revisados por pares y solo un 10\% corresponde a una Tesis Doctoral. Sobre el año en el cual se publicaron los estudios se aprecia una baja producción científica en el campo del Software Educativa orientado a cubrir las NEE en el período elegido (2004-2014) con los criterios expuestos en la sección Metodología. En los años 2008 con un $20 \%$ y 2013 un 30\%, se registra como los años de mayor publicaciones, y en el resto del rango seleccionado acumulan un 50\%. Sin embargo, en los últimos años se ha abordado con algo de intensidad el tema de la Educación Inclusiva, pero lastimosamente no hay resultados en una cantidad significativa sobre la utilización de tecnología que cubra la problemática abordada y se pueda comparar su eficiencia en el proceso de enseñanza-aprendizaje.

Sobre el tipo de diseño empleado por los investigadores en los estudios se pudo verificar que un 30\% fue Pre-experimental, un 40\% Cuasi-experimental y un 30\% Estudio de Caso; y en cuanto a las técnicas de análisis utilizadas se encontró un 20\% Prueba de Wilcoxon, 30\% T-Student, 10\% Análisis ANOVA y un 40\% Análisis Descriptivo.

En las investigaciones seleccionadas se detectó una supremacía del idioma inglés con un $70 \%$ sobre un $30 \%$ en español, relacionándose estos porcentajes con el origen de los estudios, ya que el 80\% corresponde a Europa, Asia y Norteamérica, y solo un $20 \%$ a Latinoamérica (ver figura 1).

La edad promedio de los alumnos que participaron en las investigaciones fue de 10 años, cuyo rango se encuentra ente 6 y 13 años. En cuanto al nivel académico hubo cierta variedad, con un alto porcentaje (50\%) que no correspondía a ningún nivel en específico, es decir el Software Educativo se podía utilizar en varios niveles de la escuela; un 10\% estaba dirigido para Primer Grado; 20\% para Quinto Grado y por último un $20 \%$ que no indicaban esta información, demostrando una falta de atención en el resto de niveles de la educación primaria. 


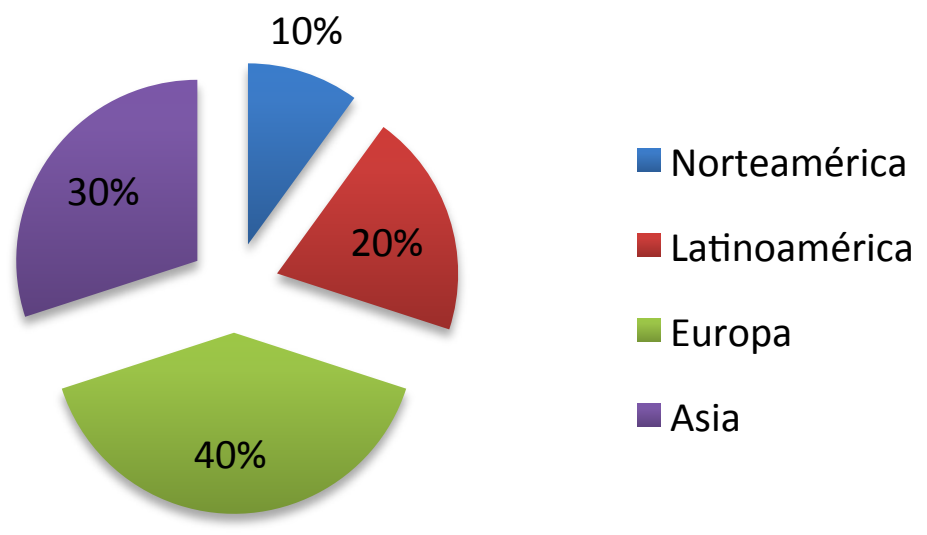

Figura 1. Diagrama de sectores que representa el origen geográfico de las investigaciones

También es importante acotar, que las muestras de los estudios fueron pequeñas, donde solo una investigación evidenció una muestra representativa que correspondió a 90 sujetos, el resto estuvo en un rango de 4 a 12. Estos datos, puede deberse a lo complejo que resulta investigar con grupos grandes de niños con Necesidades Educativas Especiales.

El área o asignatura más apoyada por los investigadores para aplicar el Software Educativo ha sido Lengua y Escritura con un 30\%, seguida de Matemáticas con el $20 \%$, y con el mismo porcentaje para la enseñanza de un Idioma Extranjero, Ciencias con el $10 \%$, y por último un $20 \%$ correspondiente al mejoramiento de la atención y la interacción social de los niños como se puede ver en la figura 2. Concluyendo con esta información, una falta de interés en el resto de áreas del conocimiento, como la Geografía, Historia y demás, asumiendo con esto que las tres primeras asignaturas citadas con mayor porcentaje representan a las áreas con mayor dificultad al momento de enseñar a los alumnos con Necesidades Educativas Especiales por parte de los docentes.

De las nueve funciones de Software Educativo (Informativa, Instructiva, Motivadora, Evaluadora, Investigadora, Expresiva, Metalingüística, Lúdica e Innovadora), que citan tanto Marqués (1995) como García-Valcárcel y Hernández (2013), solo tres han sido utilizadas en los estudios seleccionados: con un mayor porcentaje (50\%) la función instructiva, seguida de la función evaluadora (40\%) y tan solo con el 10\% la función motivadora, como se aprecia en la figura 3, evidenciado una línea amplia de investigación para un futuro inmediato.

Por otro lado, en los estudios seleccionados se registraron cierta variedad en la cantidad de tiempo que eligieron los investigadores para aplicar el Software Educativo, siendo el período con más preferencia el de cuatro a seis meses con un $40 \%$, seguido por el de uno a tres meses con el $20 \%$, y con un $10 \%$ el de siete a diez meses. Esta característica fue importante escoger en la codificación de variables, por cuanto puede ser útil para futuras investigaciones en otros contextos utilizando el mismo programa informático. 


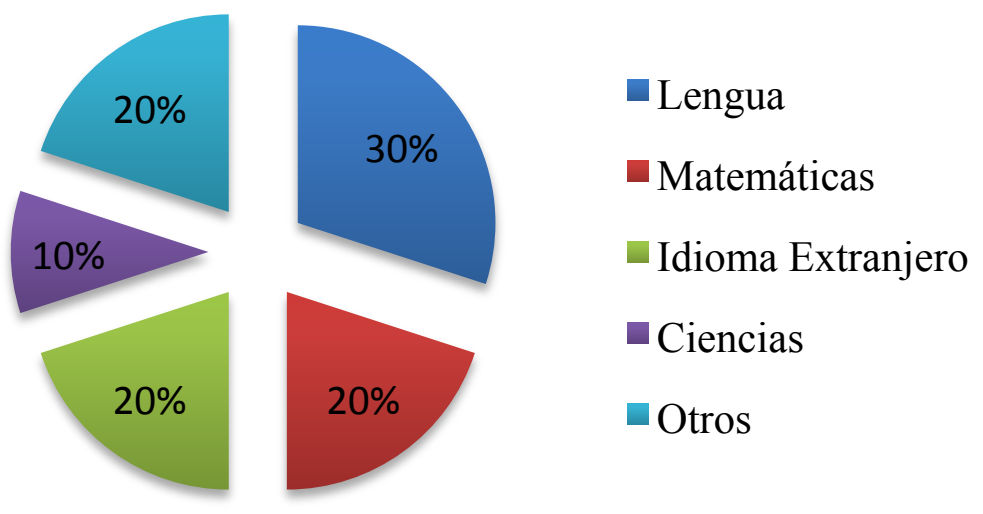

Figura 2. Diagrama de sectores que representa el área o asignatura que fue incluida en los estudios primarios.

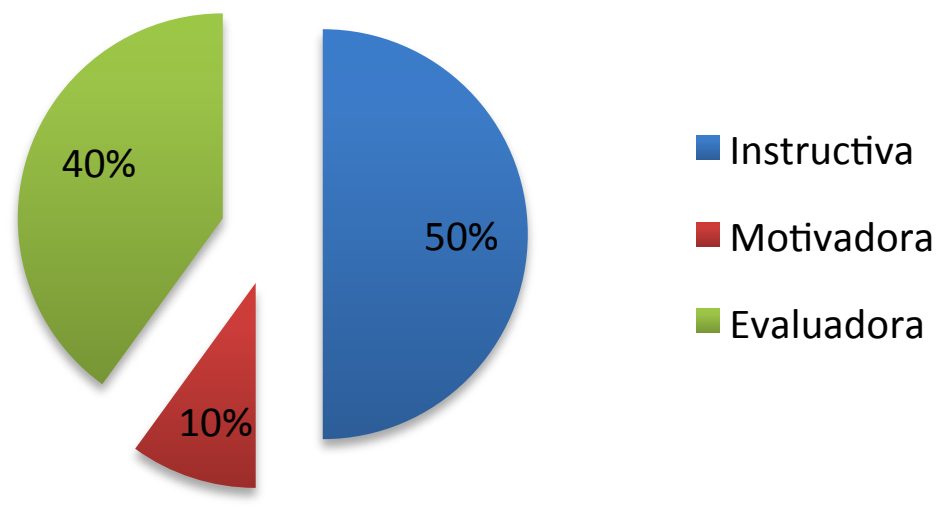

Figura 3. Diagrama de sectores que representa la función del Software Educativo utilizado en la investigación

Y por último, mencionamos las Necesidades Educativas Especiales que han sido motivo de estudio por parte de los investigadores, mediante el apoyo del Software Educativo, evidenciando una mínima atención. Solo cinco se han detectado de las descritas por Paula (2003) (Alteraciones Físicas, Deficiencias intelectuales, Alteraciones de la Personalidad, Desajustes Sociales, Perturbaciones del lenguaje, Perturbaciones psicomotrices y otras específicas escolares), con el 50\% la Discapacidad Intelectual, 20\% el Trastorno por Déficit de Atención (TDA), 20\% la Discapacidad sensorial (auditiva y visual) y un $10 \%$ para la Dislexia, como se observa en la figura 4 . Lo que conlleva a una reflexión profunda por el resto de NEE que requieren ser apoyadas para superar las Dificultades del Aprendizaje en las aulas escolares, y convertirlas en verdaderas instituciones de educación inclusiva. 


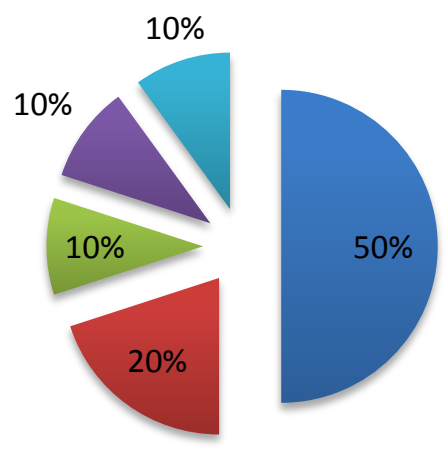

\author{
Discapacidad \\ Intelectual \\ TDA \\ Discapacidad \\ Visual \\ Discapacidad \\ Auditiva
}

Figura 4. Diagrama de sectores que representa el porcentaje de cada una de las

Necesidades Educativas atendidas en las investigaciones

\title{
Análisis descriptivo del tamaño del efecto medio
}

En este trabajo como se indicó anteriormente se transformó a una métrica común (d de Cohen) todos los resultados de los estudios seleccionados, permitiéndonos obtener los estadísticos descriptivos básicos, los mismos que representan a los 14 tamaños del efecto independientes, de los cuales se obtuvo una media $d_{+}=1.22$, con una variabilidad del 1.34 .

Para la unificación de los resultados obtenidos en los estudios primarios se aplicó la estimación combinada del tamaño de efecto con valores $d$, produciéndose unos valores que se muestran en la tabla 4, y que nos permitieron hallar la Estimación puntal del tamaño del efecto, siendo éste $\boldsymbol{d} \mathbf{=}=\mathbf{0 . 2 1}$, representando un valor algo significativo el efecto del Software Educativo en apoyo a los alumnos con Necesidades Educativas Especiales.

En cuanto al intervalo de confianza (IC del 95\%), en el cual se encuentra el valor estimado del tamaño del efecto, resultó que está entre .02 y .4, pero el valor que mejor representa es $\mathbf{d}$. $=. \mathbf{2 1}$, siendo éste un "tamaño de efecto bajo", ya que Cohen (1988) manifiesta que a partir de .80 se puede considerar un "tamaño de efecto alto". Además, se puede deducir que un contraste sobre la hipótesis nula (tamaño del efecto es nulo $\delta=0$ ) basado en el conjunto de estudios seleccionados, con un nivel de significación de $\alpha=.05$, nos conduce a rechazar $\mathrm{H}_{0}$, dado que el intervalo descrito (.02 - .4) no incluye el valor de 0 . Por tanto, se concluye que existe una asociación entre las variables Software Educativo y las Necesidades Educativas Especiales.

Sobre la dispersión de los resultados que mostraron los estudios primarios, se concluyó que hay homogeneidad, es decir que la variabilidad del tamaño del efecto del Software Educativo aplicado en las Necesidades Educativas de los estudios seleccionados es estadísticamente igual, ya que mediante las fórmulas propuestas por Hedges y Olkin (1985) tomadas de Botella y Gambara (2002) se obtuvo que Qt $=16.82$ es menor del valor tabular ${ }_{0.95}^{2}(\mathrm{k} 13)=22.36$, siendo estos resultados concluyentes para rechazar la hipótesis nula $\left(\mathrm{H}_{0}\right)$. 
Tabla 4

Integración de resultados

\begin{tabular}{cccccccc}
\hline No. Estudio & $\mathbf{d}_{\mathbf{i}}$ & $\mathbf{S}_{\mathbf{d}}{ }^{2}$ & $\mathbf{N}_{\mathbf{1}}$ & $\mathbf{N}_{\mathbf{2}}$ & $\mathbf{w}_{\mathbf{i}}$ & $\mathbf{w}_{\mathbf{i}}{ }^{*} \mathbf{d}_{\mathbf{i}}$ & $\mathbf{W}_{\mathbf{i}}{ }^{*} \mathbf{d}_{\mathbf{i}}{ }^{2}$ \\
\hline 1 & 4.33 & 153.47 & 12 & - & .007 & .03 & .13 \\
2.1 & 2.69 & 4.80 & 9 & - & .21 & .56 & 1.51 \\
2.2 & 1.92 & 10.91 & 9 & - & .09 & .17 & .33 \\
3 & 2.03 & .9 & 7 & - & 1.11 & 2.25 & 4.57 \\
4 & 1.45 & 18.85 & 7 & - & .05 & .07 & .11 \\
5.1 & .15 & - & 90 & 90 & 44.87 & 6.73 & 1.01 \\
5.2 & .05 & - & 90 & 90 & 44.99 & 2.25 & .11 \\
6 & .70 & - & 9 & 9 & 4.24 & 2.97 & 2.07 \\
7.1 & -.41 & 9.34 & 4 & - & .11 & -.05 & .02 \\
7.2 & .58 & 2.94 & 4 & - & .34 & .19 & .11 \\
8 & 2.46 & - & 9 & 8 & 2.41 & 5.92 & 14.58 \\
9.1 & .28 & 3.45 & 7 & - & .29 & .08 & .02 \\
9.2 & 1.11 & 2.83 & 7 & - & .35 & .39 & .43 \\
10 & -.20 & 1.44 & 5 & - & .69 & -.14 & .03 \\
& & $\boldsymbol{\Sigma}$ & & & 99.76 & 21.42 & 25.03 \\
\hline
\end{tabular}

Y, finalmente se calculó el Binomial Effect Size Display (Expresión Binomial del Tamaño del Efecto), propuesto por Rosenthal y Rubin (1982) citado por Botella y Gambara (2002), para comprender mejor los resultados obtenidos en el meta-análisis, indicándonos que los alumnos del Grupo Experimental mejoraron un $72 \%$ al aplicar el Software Educativo y los del Grupo de Control un $28 \%$.

\section{Discusión y conclusiones}

El desarrollo del presente trabajo ha permitido valorar aún más la importancia que tienen en la actualidad las Tecnologías de la Información y Comunicación en el proceso de enseñanza-aprendizaje, y sobre todo cuando lo abordamos en el tema de superar las dificultades de aprendizaje que se generan en el salón de clase. Por ello el objetivo principal fue determinar el efecto que produce el Software Educativo en la atención de las Necesidades Educativas Especiales en niños de 6 a 12 años correspondiente a la Educación Primaria, para concienciar a todos los entes responsables de generar una verdadera Escuela Inclusiva, sobre todo al profesorado, ya que hay gran cantidad de recursos diseñados, esperando ser utilizados en la práctica educativa.

En cuanto a las Necesidades Educativas Especiales atendidas por los investigadores en los estudios primarios, la Discapacidad Intelectual fue la que más apopo tuvo con un 50\%, seguido del Trastorno por Déficit de Atención con el 20\%, la Dislexia, la Discapacidad Visual y Auditiva con un 10\% cada una, lo que conlleva a una reflexión 
sobre la falta de atención al resto de Necesidades existentes, coincidiendo en parte estos resultados con lo que menciona Doval (2011) sobre las Tecnologías Adaptadas a la Diversidad que más se utilizan en la atención a alumnos con NEE.

El área o asignatura que escogieron los investigadores para aplicar el Software Educativo con un mayor porcentaje fue Lengua y Escritura con un 30\%, seguido de Matemáticas y Lengua Extranjera con un $20 \%$ cada una, Ciencias con el $10 \%$ y un $20 \%$ para actividades de motivación y atención, confirmándose con estos resultados la atención en las dificultades que se presentan en enseñar los números y las letras, ya que este tipo de material se ha ido masificando, pero lastimosamente no hay suficientes investigaciones que garanticen su uso y efectividad. En este punto también hay que añadir, el poco alcance que han tenido las investigaciones en cuanto a la aplicación del Software Educativo en los diferentes niveles de la escuela, ya que solo el 10\% estaba dirigido a Primer Grado y un 20\% para Quinto Grado, y el resto estuvo orientado a todos los grados (niveles) o no especificaron.

Por esta razón, se justifica de alguna manera la elección de los investigadores en cuanto a la Función del Software Educativo, ya que el 50\% corresponde a la Instructiva, seguido de la Función Evaluadora con un $40 \%$ y solo un 10\% a la motivadora, dejando un gran vacío en el resto de Funciones que se detallaron en el Marco Teórico.

Por otro lado, la estimación del tamaño del efecto conjunto (.21), tiene una significación baja con respecto al valor 80 que estableció Cohen (1988) como "tamaño de efecto alto", pero de alguna manera vale manifestar la eficacia que resulta utilizar el Software Educativo como medio didáctico en apoyo a las NEE de los niños. Esta significación se puede confirmar con la valoración que se realizó con el Binomial del tamaño del Efecto, ya que al Grupo que se le facilitó el Software Educativo para su uso alcanzan un mejoramiento del $72 \%$ frente a un $28 \%$ del Grupo que no lo utilizó.

Sobre la Hipótesis planteada para establecer el grado de Heterogeneidad de los resultados de los estudios, se obtuvo en el estadístico Qt un valor de 16.82, el cual resultó menor que el valor tabular ${ }_{0.95} \chi^{2}(\mathrm{k} 13)=22.36$, que significó rechazar la Hipótesis Nula. De esta manera se concluyó que hay homogeneidad, y no es necesario el análisis inferencial.

Con relación a las limitaciones del presente Meta-análisis se manifiesta en el sesgo de publicación de los estudios, ya que la muestra de investigaciones seleccionadas es pequeña, con relación a las bases de datos ubicadas en el procedimiento de búsqueda, lo cual refleja la falta de resultados en las investigaciones que diseñaron e implementaron algún Software Educativo. Además, se puede manifestar que no se consiguió realizar un análisis estadístico más exhaustivo por observar diferencias en las Técnicas de Análisis, en los Software (programas informáticos) utilizados, la variedad de asignaturas atendidas, y por supuesto la diversidad de Necesidades Educativas Especiales detectadas, por tal motivo se determinó de forma general el tamaño del efecto del Software Educativo en apoyo a las Necesidades Educativas Especiales como variables independiente y dependiente respectivamente.

Y por último, indicar que una meta de este trabajo era plantear nuevas líneas de investigación sobre el tema de las TIC en el proceso de enseñanza-aprendizaje, sobre todo para subsanar en parte las NEE que tienen los alumnos, por ello para futuros estudios se pueden plantear meta-análisis con respecto a las APP (Programas con 
características especiales), sobre todo para recursos móviles. Existe la necesidad de concienciar a los investigadores para continuar en la tarea de innovar recursos didácticos específicos para las Necesidades Educativas Especiales y para cada área del conocimiento, cumpliendo con la "Escuela para todos", como señalan la UNESCO y el Ministerio de Educación y Ciencia (MEC) (1994). Y, por qué no, pensar a corto o mediano plazo el desarrollar recursos que cumplan los principios del diseño universal.

\section{Referencias}

Las referencias marcadas con un asterisco $\left(^{*}\right)$ indican los estudios que se incluyeron en el meta-análisis.

*Alcaraz, S., Ballesta, J., \& Lozano, J. (2011). Software para enseñar emociones al alumnado con trastorno del espectro autista. Revista Científica Educomunicación, 18(36), 139-148. doi:10.3916/C36-2011-03-05

Amar, M. (2013). Educación y TIC en la sociedad del conocimiento. En J. Sánchez \& J. Ruiz (Eds.), Recursos didácticos y tecnológicos en educación (pp. 15-23). Madrid: Editorial Síntesis.

ARARTEKO (2001). La respuesta a las necesidades educativas especiales en la CAPV. Bilbao: Ararteko.

*Atkins, A., Chakpitak, N., Sharp, B., Sureephong, P., \& Wicha, S. (2012). An animated dictionary for hearing-impaired students in Thailand. Journal Research in Special Educational Needs, 12, 234-244. doi:10.1111/j.1471-3802.2012.01239.x

Bautista, R. (2002). Educación Especial y Reforma Educativa. En R. Bautista (Ed.), Necesidades educativas especiales (pp. 19-29). Archidona Málaga: Aljibe.

Boletín Oficial del Estado (2006). Ley Orgánica de Educación (No. 106). Recuperado de https://www.boe.es/buscar/pdf/2006/BOE-A-2006-7899-consolidado.pdf

Botella, J., \& Gambara, H. (2002). Qué es el meta-análisis. Madrid: Editorial Biblioteca Nueva S.L.

Cabero, J. (2008). Reflexiones sobre las tecnologías como instrumentos culturales. En F. Martínez \& M. Prendes (Eds.), Nuevas Tecnologías y Educación (pp. 15-19). Madrid: Pearson Prentice Hall.

Cabero, J., \& Córdoba, M. (2009). Inclusión educativa: inclusión digital. Revista Educación Inclusiva, 2(1), 61-77. Recuperado de http://dialnet.unirioja.es/descarga/ articulo/3011853.pdf

Cabero, J., Fernández, J., \& Córdoba, M. (2007). Las TIC como elementos en la atención a la diversidad. En J. Cabero, M. Córdoba \& J. Fernández (Eds.), Las TIC para la igualdad (pp. 15-35). Sevilla: Editorial MAD.

Calvo, I., \& Verdugo, M. (2012). Educación inclusiva, ¿una realidad o un ideal?. Revista Edetania: Estudios y propuestas socio-educativas, 41, 17-30. Recuperado de https://dialnet.unirioja.es/servlet/articulo?codigo $=4089625$

Cebrián, M. (2011). Los centros educativos en la sociedad de la información y el conocimiento. En M. Cebrián \& M. Gallego (Eds.), Procesos educativos con TIC en la sociedad del conocimiento (pp. 23-31). Madrid: Pirámide.

Cerdá, M. (1990). Niños con necesidades educativas especiales: Bases conceptuales, diagnósticas y tratamiento. Valencia: Promolibro. 
Cohen, J. (1988). Statistical power analysis for the behavioral. New Jersey: Erlbaum.

${ }^{*}$ Contreras, E., \& Contreras I. (2011). Desarrollo de habilidades académicas funcionales en niños con discapacidad intelectual mediante software educativo. En J. Acevedo, M. Trujillo \& M. López (Eds), La problemática de los grupos vulnerables: Visiones de la realidad (105-127). Recuperado de http://www.ts.ucr.ac.cr/binarios/libros/libros-000023.pdf

Cortés, C. (1999). Necesidades educativas especiales en la ESO: Guía para la respuesta educativa a las necesidades del alumnado con discapacidad psíquica. Pamplona: Gobierno de Navarra, Departamento de Educación y Cultura.

${ }^{*}$ Cullen, J., Richards S., \& Lawless C. (2008). Using Software to Enhance the writing skills of students with Special Needs. Journal of Special Education Technology, 23(2), 33-44. Recuperado de http://jennifercullen.weebly.com/uploads/1/0/8/3/10835528/ jset_article.pdf

Doval, M. (2011). Tecnologías de apoyo a la diversidad en la escuela inclusiva. En M. Cebrián \& M. Gallego (Eds.), Procesos educativos con TIC en la sociedad del conocimiento (pp. 45-57). Madrid: Pirámide.

Durlak J., \& Lipsey M. (1991). A Practitioner's guide to meta-analysis. American Journal of Community Psychology, 19(3), 291-332. Recuperado de http://link.springer.com/ article /10.1007/BF00938026

García-Valcárcel, A. (2003). Tecnología Educativa: Implicaciones educativas del desarrollo tecnológico. Madrid: Editorial La Muralla.

García-Valcárcel, A., \& Hernández, A. (2013). Recursos tecnológicos para la enseñanza e innovación educativa: Las tecnologías de la información y la comunicación en el contexto educativo actual. Madrid: Editorial Síntesis.

Glass, G. (1976). Primary, secondary, and meta-analysis. Educational Researcher, 5(10), 3-8. Recuperado de http://www.jstor.org/stable/1174772?seq=1\#page_scan_tab_contents

*Gómez, C. \& Ortega, J. (2006). Computer-assisted teaching and mathematical learning in Down Syndrome children. Journal of Computer Assisted Learning, 22(4), 298-307. doi:10.1111/j.1365-2729.2006.00179.x

González, A., \& De Pablos, J. (2015). Factores que dificultan la integración de las TIC en las aulas. Revista de Investigación Educativa, 33(2), 401-417. doi: 10.6018/rie.33.2.198161

González, E. (1996). La Educación Especial: Conceptos y datos históricos. En E. González (Ed.), Necesidades educativas especiales: Intervención psicoeducativa (pp. 15-44). Madrid: CCS.

Hernández, M. (2009). Estrategias de búsqueda de información para la generación de conocimiento en la Red. (Tesis doctoral). Recuperada de http://gredos.usal.es/jspui/ handle/10366/76265

Hervás, C., \& Toledo, P. (2007). Las nuevas tecnologías como apoyo a los sujetos con necesidades educativas especiales. En J. Cabero (Ed.), Nuevas Tecnologías Aplicadas a la Educación (pp. 279-291). Madrid: McGraw-Hill/Interamericana de España.

*Hetzroni, O., \& Tannous, J. (2004). Effects of a Computer-Based Intervention program on the communicative functions of children with Autism. Journal of Autism and Develomental Disorders, 34(2), 95-113. Recuperado de http://ftp.aspires-relationships.com/ Effects_of_a_Computer_Based_Intervention_Program_on_the.pdf 
*Jorge Ventura, M. (2012). Las Tic en el desarrollo de las competencias (meta) lingüísticas de los niños con perturbaciones en el desarrollo del lenguaje. (Tesis doctoral). Recuperada de http://dehesa.unex.es:8080/xmlui/handle/10662/377

*Jun Tan, J., \& Mei Lin, N. (2012). Hypersmart Kids: A case study on the Response of students with Dislexia and ADHD to Educational Software Games in English Language Learning. Internacional Conference "ICT for Language Learning". Recuperado de http://conference.pixel-online.net/ICT4LL2012/common/download/Paper_pdf/228IBT91-FP-Tan-ICT2012.pdf

*Lipsey, M. (1994). Identifying potentially interesting variables and analysis oppotunities. En H. Cooper \& L. Hedges (Eds), The Handbook of Research Synthesis (pp. 111-123). New York: Russel Sage Foundation.

López, I., \& Valenzuela, G. (2015). Niños y adolescentes con necesidades educativas. Revista Médica Clínica Las Condes, 26(1), 42-51. Recuperado de http://www.sciencedirect.com/science/article/pii/S0716864015000085

Luque, D., \& Rodríguez, G. (2009). Tecnología de la Información y Comunicación aplicada al alumnado con discapacidad: un acercamiento docente. Revista Iberoamericana de Educación, 49(3), 1-8. Recuperado de http://www.rieoei.org/deloslectores/ 2806Parrav2.pdf

Marchesi, Á. (2014). Del lenguaje de la deficiencia a las escuelas inclusivas. En Á. Marchesi, G. Palacios \& C. Coll (comp.), Desarrollo psicológico y educación: 3. Trastornos del desarrollo y necesidades educativas especiales (pp. 21-43). Madrid: Larousse - Alianza Editorial.

Marín, M. (2004). Alumnos con necesidades educativas especiales. San José, Costa Rica: Euned.

Marín, V., \& Latorre, M. (2007). Superar las barreras de aprendizaje en necesidades educativas especiales mediante la utilización de las TICs. Revista de Educación XX1, 9, 267-275. Recuperado de http://rabida.uhu.es/dspace/bitstream/handle/10272/2100/ b15175996.pdf?sequence $=1$

Marqués, P. (1995). Software educativo: Guía de uso y metodología de diseño. Barcelona: EMA-Estudis, S.L.

Parra, C. (2010). Educación Inclusiva: Un modelo de educación para todos. Revista Inclusión Social y Equidad en la Educación Superior, 8, 73-84. Recuperado de https:// dialnet.unirioja.es/ejemplar/290991

Paula, I. (2003). Educación Especial: Técnicas de intervención. Madrid: McGraw-Hill.

Ricoy, M. \& Feliz, T. (2002). Consideraciones previas sobre las necesidades educativas especiales. En J. González (Ed.), Necesidades educativas especiales e intervención psicopedagógica (pp. 3-30). Alcalá de Henares: Universidad de Alcalá.

*Sánchez, J., \& Elías, M. (2007). Science Learning by Blind Children through AudioBase Interactive Software. En B. K. Wiederhold, S. Bouchard, \& G. Riva (Eds.), Anual Review of Cyber Therapy and Telemedicine (vol.5, pp.157-163). San Diego, CA, United States of America: Interactive Media Institute

Sánchez J., Olivares J., \& Rosa A. (1998). Meta-análisis de las intervenciones conductuales en el tratamiento de la diabetes en España. Revista de Psicología General y Aplicada, 51(3), 501-516. Recuperado de http://dialnet.unirioja.es/servlet/articulo? codigo $=2498070$ 
Sanz del Río, S. (1996). Integración de alumnos con necesidades educativas especiales: Panorama internacional. Madrid: Real Patronato de Prevención y de Atención a Personas con Minusvalía.

*Simion, E., \& Folostina, R. (2013). Integrated learning systems and their effects on attention of children with intelectual disability. En I. Roceanu (Ed.), $9^{\text {th }}$ Internacional Conference eLearning and Software for Education, (vol. 2, pp. 604-509). Bucharest: Editura Universitatii Nationale de Aparare "Carol I". doi: 10.12753/2066-026X-13-206

Organización de las Naciones Unidas para la Educación, la Ciencia y la Cultura [UNESCO] y Ministerio de Educación y Ciencia [MEC] (1994). Declaración de Salamanca y Marco de Acción. Salamanca: UNESCO.

Organización de las Naciones Unidas para la Educación, la Ciencia y la Cultura [UNESCO] (2008). La educación inclusiva: El camino hacia el futuro (No. 48/3). Recuperado de http://www.ibe.unesco.org/fileadmin/user_upload/Policy_Dialogue/48th_ ICE/CONFINTED_48-3_Spanish.pdf

Fecha de recepción: 23 de octubre de 2015

Fecha de revisión: 23 de octubre de 2015

Fecha de aceptación: 20 de mayo de 2016 